\title{
Autism Spectrum Disorder in Adult Patients: Clinical Presentation And Management
}

\author{
Maxym Konyushok
}

\begin{abstract}
Autism is a developmental disorder, neurological in nature, that affects a person's thinking, perception, attention, social skills, and behavior. Majority of researches and descriptions of autism are devoted to diagnosing children and the impact of autism on developmental goals (e.g., peer play, learning skills and family relationships). Although the symptoms of autism do not change during adulthood, the various manifestations of autism become less or more important as a result of changes in adult daily functioning. It is not always easy to understand exactly how such complex disorder affects the lives of adults. Although every person with autism has their own set of symptoms (and they can vary in severity), there are certain signs of autism that are considered the most typical for the disease. Autism in adults can be presented in different ways, depending on the form of the disease. In order for an adult with autism to adapt to the surrounding environment as best as possible, rehabilitation is necessary. The earlier the process begins, the greater the results can be achieved, so ideally it starts as soon as autism is diagnosed. The prognosis for each person with autism depends on how the disease manifests.
\end{abstract}

Index Terms - autism spectrum disorder, ASD, child psychiatry, autism spectrum disorder in adult, antipsychotic, ADHD, psychopharmacology

\section{INTRODUCTION}

While autism is considered a childhood disorder, its dramatically increasing prevalence due to the absence of effective therapy and means that it is becoming an adult disorder. At the same time, less than $2 \%$ of studies are devoted to the study of adults with the autism spectrum disorder (ASD) [1]. Children and adults with ASD show a significant incidence of comorbid depression, anxiety disorder, bipolar disorder, obsessive-compulsive disorders, psychosis, self-harm and somatic problems [2]-[7]. Some authors believed that diagnosed comorbid mental disorders such as anxiety, obsessive-compulsive disorder (OCD) and attention deficit hyperactivity disorder (ADHD) are more common than the others [8]-[11]. Some authors reported that psychotic disorders uncommon for ASD [8].

In contrast, some authors estimated that the comorbidity of ASD with psychosis is $28 \%$ and identified features of atypical presentation required for the diagnosis of schizophrenia that include shorter duration, more acute and transient course, and the presence of affective disorders [12]. In $42 \%$ of the investigated patients, there is an imperceptible decrease in the level of functioning. $32 \%$ of the patients are diagnosed with

Published on August 17, 2020.

Maxym Konyushok, Therapeutic and Preventive Center of Mental Health "Stozhar", Ukraine.

(corresponding e-mail: maxymkonyushok@ ${ }^{@}$ gmail.com) unspecified psychosis and $21 \%$ are diagnosed with schizophrenia. The diagnosis of schizophrenia is difficult, so psychiatrists usually do not rush to make a diagnosis and observing the patient for at least six months [13], [14]. During this period, psychiatrists repeatedly evaluate the patient who is suspected of schizophrenia. ASD and schizophrenia have significant similarity [12], [15].

The relationship between autism and catatonia is also a subject of controversy among researchers. Catatonia is divided into catatonic stupor and catatonic excitement that develops in 17\% of adolescents and young adults with ASD [16]. A known theory proposes that in some cases, autism is a childhood manifestation of "periodic catatonia" [17]. The similarity of movement disorders in autism and catatonia is striking.

Some researchers pay attention to the small number of studies of aggressive behavior in adults with ASD [18]. The frequency of aggressive behavior among low-functioning adolescents with ASD was 58\% (according to caregivers) and $38 \%$ (according to parents) [19]. The authors noted the connection between aggression and anxiety in adolescents with autism and the importance of behavioral predictors of aggression: agitation, sweating, screaming and excitement.

\section{Clinical PRESENTATION AND TRAits OF ASD IN ADULTS}

Among the syndromes presenting in adult ASD pedagogical neglect, speech underdevelopment and emotional deficit are prevailed. In general, patients, even in psychotic states, often demonstrate childish dependence on loved ones and a high degree of suggestibility. Psychoses may be developed in a third of the patients. Clinically, they are predominantly catatonic and in isolated cases - delusional with hallucinations. The psychopathology of ASD in adults gravitated towards a mixed affective-catatonic presentation

The diagnostic qualifications of various psychopathological conditions in adults with ASD and some aspects of therapy raise many questions and require further development. The analysis of mentioned in the introduction literature showed the persistence of ASD symptoms throughout the life in most patients with significant pedagogical neglect, speech underdevelopment and chronic catatonia [1], [2], [8], [12], [16], [18], [19].

In general, in one third of patients the progress of the disease is stable from childhood to adulthood without a new 
psychopathology or an obvious deepening of the emotional deficit. It seems that it is precisely for such patients that the diagnosis of ASD in adulthood is most justified. Among these patients there are several cases, similar in phenotypic and clinical manifestations with the following features: hypertelorism, relatively wide mouth, high forehead, massive lower jaw, pedantry, loud, sometimes chanting, speech, general tension and sharp deficit of positive emotions.

The rest of adult ASD patients, presents with different syndromes and different dynamics, most often to be presented with mood (affective) and catatonic symptoms. The comorbidity of catatonia, mood symptoms and psychotic symptoms is reported [16], [20], [21]. Some authors reported the information about the unity of catatonia, mood disorders and psychotic symptoms with ASD [8], [12], [22].

Evidence of the validity of the diagnosis of schizophrenia is the detection of signs of the course of the disorder with an increase in emotional deficit and the appearance of typical schizophrenic symptoms. In this part of the diagnosis in patients with ASD the difficulties associated with the early onset of the disorder, possible course of the disorder on the positive dynamics of child development, the slow growth of negative symptoms and the non-verbal or low verbal nature of a large percentage of the ASD patients, making a clinical assessment of thought disorders extremely embarrassing. Only in isolated cases, patient's parents may note a significant increase in indifference and inactivity. The study of formal thought disorders in speaking adults with ASD, with the possible detection or non-detection of typical schizophrenic disorders, requires further close study. The same difficulties arise with the assessment of the chronic catatonic symptoms in terms of its delimitation from the clinic of childhood ASD.

Despite the diagnostic difficulties, the assessment of the dynamics of ASD in adulthood with the frequent addition of the entire spectrum of psychiatric disorders raises the assumption that the ASD belongs to the schizophrenia spectrum disorder in most cases (at least two thirds of the patients), and in a quarter of cases, the diagnosis of catatonic schizophrenia may be fully justified. Cases of paranoid schizophrenia are presented rarely.

Epilepsy is a brain disorder characterized by repeated seizures or convulsions. Epilepsy is much more common in people with autism than in the general population [23]. Seizures are more common in people with less developed verbal abilities. Studies show that 15-30 percent of people with autism also suffer from epilepsy. About 5 percent of people who develop epilepsy in early childhood also develop autism [23]-[25].

Depression is not just a constant decline in mood, pessimism, and a desire for self-isolation [6]-[9]. It is a mental disorder that has serious consequences. The patient loses the ability to enjoy life, he is not given the solution of difficult problems, is prone to negative ways out of any situation. Depression is 4 times more common in ASD people than in healthy people, but experts still have little understanding of its causes and the best approaches to diagnosis and treatment [26].

Bipolar disorder is associated with severe emotional swings [3], [4], [27]. Elevated and depressed mood is associated with significant changes in how energetic a patient is, how active patient is, and how much need for sleep is required. There is high incidence of bipolar disorder among family members of patients with ASD [28]. The bipolar disorder may be comorbid with the high-functioning ASD [29]. The ASD may share common vulnerability genes with the bipolar spectrum.

Due to the prevalence, social and medical consequences, the pathological attraction to gambling attracts great attention [30]. At the same time, it belongs to one of the prominent places in the studied concept of the disorder of addictive behavior. ASD patients are more prone to addiction to computer games, which can be associated with aggression and other problems [31]. People with ASD may find video games very engaging because they provide immediate reward, visually stimulate, and avoid face-to-face communication or social interaction. Scientists found that video games, especially some of their varieties, were associated with problematic oppositional behaviors such as arguing and aggression.

Stroke is an acute disorder of cerebral circulation, the symptoms of which persist for more than one day [32]. There is no documented links between ASD and stroke. However, one in three ASD patient is obese, compared with $13 \%$ of non-affected people [33]. A number of factors contribute to this. The factors include a tendency to avoid physical activity and poor diet. In addition, heavy weight gain can be a common side effect of some drugs that may be prescribed to correct problem behaviors in autism. It is important to get ASD patient to be checked out as there are a number of medical problems and illnesses that can lead to being overweight. One of the most serious diseases is stroke. Although these metabolic disorders are relatively rare, these are very serious problems that are important to diagnose on time. It is very important to rule out these conditions before making a weight management plan. Lipid profile testing (LPT) can help prevent stroke in people with ASD. LPT is a set of specific blood tests to determine deviations in the body's fat metabolism, which is of great importance for the diagnosis of atherosclerosis.

Poor oral hygiene has been proven to lead to periodontal disease and multiple caries [34]. Caries and its complications, periodontal diseases, dentoalveolar anomalies, delayed eruption of permanent dentition and systemic enamel hypoplasia are most common in people with ASD [35]. People also have problems with bite as a result of bad habits: sucking fingers, biting nails and foreign objects. Accordingly, they need to more often seek dental and orthodontic care. Muscle tone is weakened, coordination of movement is impaired, and salivation is increased. Such patients adhere to a certain diet, eat mostly soft and sweet foods; due to the impaired coordination of the tongue, they usually keep it in their mouth for a long time before swallowing food. All this increases the predisposition to the development of caries.

\section{Treatment StRategy In Adult ASD}

Adults with ASD do not make eye contact and are in their thoughts. They can, without ceasing, make various senseless movements, arrange objects and things in a certain sequence that is only understandable to them. It is often accompanied by obsessions, during which they become indifferent to the 
world around them. Only something unexpected and unusual can bring them out of this state. Often people with autism have seizures with unpredictable consequences and they should be adequately managed. Almost half of autistic people are known to have self-injury. Such patients require constant care, attention, since in a severe form of the disease they cannot serve themselves.

The issue of the optimal management of patients with ASD deserves special attention. The presence of the resistant to therapy symptoms of ASD and chronic catatonia is an evidence of inappropriately prescribed treatment that will be doomed in advance to failure. Adults with stable clinical presentation of ASD are more in need of comprehensive social and psychological interventions and lifelong social support than pharmacological intervention. Patient admissions should be carried out with the actual presence of social indications and ineffectiveness of outpatient treatment, and its duration should preferably be reduced to as shorter as possible. Taking detailed history especially focusing on triggers of worsening of symptoms will help clinicians to distinguish an actual relapse from the situational response of a chronic psychotic patient.

It should be noted that certain technical difficulties may be faced during adult ASD treatment. Not all patients may agree to come for a face-to-face consultation for various reasons (e.g., long distance or unwillingness to visit a psychiatrist to avoid stigmatization). Collateral history from caregivers or parents may help in diagnosis and treatment. Some of them may reflect observation and objectivity of clinical presentation, but some may deny possible disorders and present the condition of the guardian in a more favorable light.

Patients with ASD need help at any age because even the symptoms of adult autism do not go away over time. Unfortunately, patients with severe forms of pathology need daily care and medical supervision throughout their lives. This is a difficult burden for parents, but a properly selected correction program can make life easier for loved ones and develop basic skills in an autistic person.

Increasing adaptive skills makes the everyday life of autistic people much easier. When they understand themselves well and their place in society, it is easier for them to make verbal and non-verbal contact, they begin to live fully, even taking into account the specifics of the disorder. Behavioral psychotherapy provides similar assistance, but it is not just a specialist who can help the autistic adult.

Treatment in adults includes overcoming shyness, helping with socialization, and stopping the progression of the disease. It is necessary to minimize the frequency of the patient's attacks. Treatment will directly depend on the symptoms and course of the disease. The sooner the treatment started, the best results can be achieved.

\section{CONCLUSIONS}

Autism spectrum disorder persists into adulthood, but few doctors know how to recognize and diagnose autism in adults. ASD is a spectrum disorder, meaning its symptoms can range from mild to severe. There is no laboratory test to determine if a person has autism, so it is diagnosed based on behavior and observation. The symptoms of ASD manifest themselves differently in every person. This is why it is so difficult to diagnose ASD in adults. In addition, many doctors are familiar with the symptoms of autism in children, but not in adults. Behavior should be considered holistically, all symptoms and manifestations should be taken into account, and not diagnosed based on one or two symptoms and ignoring others. ASD patients should be provided with daily personal and social support and medical care throughout their life. Milder degree of ASD needs social activities, in which, they can learn social norms - for example, greet each other when they meet, nod their heads in agreement, learn to express their feelings and not be afraid of strangers. Since many autistic people are able to work, they should be taught communication skills and be supported with employment. Psychopharmacological interventions usually are not effective at all and should only be considered for a short time.

\section{REFERENCES}

[1] Smith, L. E., Greenberg, J. S., \& Mailick, M. R. (2012). Adults with autism: Outcomes, family effects, and the multi-family group psychoeducation model. Current psychiatry reports, 14(6), 732-738.

[2] Murphy, C. M., Wilson, C. E., Robertson, D. M., Ecker, C., Daly, E. M., Hammond, N., ... \& McAlonan, G. M. (2016). Autism spectrum disorder in adults: diagnosis, management, and health services development. Neuropsychiatric disease and treatment.

[3] Tsarkov, A., \& Petlovanyi, P. (2016). Bipolar Disorder in Child Psychiatric Practice: A Case Report. Medical Journal of Zambia, 43(1), 41-46.

[4] Tsarkov, A., \& Petlovanyi, P. (2016). Bipolar disorder in child psychiatric practice.

[5] Leyfer, O. T., Folstein, S. E., Bacalman, S., Davis, N. O., Dinh, E., Morgan, J., ... \& Lainhart, J. E. (2006). Comorbid psychiatric disorders in children with autism: interview development and rates of disorders. Journal of autism and developmental disorders, 36(7), 849-861.

[6] Tsarkov, A., \& Petlovanyi, P. Depressive Disorder in Child Psychiatric Practice: A. The Health Press, 9.

[7] Petlovanyi, P., \& Tsarkov, A. (2017). Depressive Disorder in Child Psychiatric Practice: A Case Report. Health Press Zambia Bull, 1(5), 9.

[8] Larson, F. V., Wagner, A. P., Jones, P. B., Tantam, D., Lai, M. C., Baron-Cohen, S., \& Holland, A. J. (2017). Psychosis in autism: comparison of the features of both conditions in a dually affected cohort. The British Journal of Psychiatry, 210(4), 269-275.

[9] Petlovanyi, P., \& Tsarkov, A. (2020). Practical guide and some recommendations for the diagnosis and management of Attention deficit hyperactivity disorder (ADHD). World Journal of Advanced Research and Reviews, 6(3), 257-261.

[10] Anatolii, T., Patrick, M., \& Petro, P. (2020). Practical guide and some recommendations for the diagnosis and management of Attention deficit hyperactivity disorder (ADHD). World Journal of Advanced Research and Reviews, 6(3), 257-261.

[11] Tsarkov, A., \& Petlovanyi, P. (2017). Omega-3 Fatty Acids as an Alternative Treatment for Children with Attention Deficit Hyperactivity Disorder. Imperial Journal of Interdisciplinary Research (IJIR), 3, 1378-1380.

[12] Edition, F. (2013). Diagnostic and statistical manual of mental disorders. Am Psychiatric Assoc.

[13] Anatolii, T., Patrick, M., \& Petro, P. (2020). Uncommon presentation: Folie à deux (Case study). World Journal of Advanced Research and Reviews, 6(3), 043-049.

[14] Tsarkov, A., Msoni, P., \& Petlovanyi, P. (2020). Uncommon presentation: Folie à deux (Case study). World Journal of Advanced Research and Reviews, 6(3), 043-049.

[15] Petlovanyi, P., \& Tsarkov, A. Child Schizophrenia: Theory and Practice.

[16] Wing, L., \& Shah, A. (2000). Catatonia in autistic spectrum disorders The British Journal of Psychiatry, 176(4), 357-362.

[17] Breen, J., \& Hare, D. J. (2017). The nature and prevalence of catatonic symptoms in young people with autism. Journal of Intellectual Disability Research, 61(6), 580-593.

[18] Dhossche, D. M. (2004). Autism as early expression of catatonia Medical Science Monitor, 10(3), RA31-RA39. 
[19] Matson, J. L., Sipes, M., Fodstad, J. C., \& Fitzgerald, M. E. (2011). Issues in the management of challenging behaviours of adults with autism spectrum disorder. CNS drugs, 25(7), 597-606.

[20] Tsai, L. Y. (2013). Asperger's disorder will be back. Journal of autism and developmental disorders, 43(12), 2914-2942.

[21] Dell'Osso, L., Dalle Luche, R., Gesi, C., Moroni, I., Carmassi, C., \& Maj, M. (2016). From Asperger's autistischen psychopathen to DSM-5 autism spectrum disorder and beyond: a subthreshold autism spectrum model. Clinical practice and epidemiology in mental health: CP \& $\mathrm{EMH}, 12,120$.

[22] Kolvin, I. (1971). Studies in the childhood psychoses I. Diagnostic criteria and classification. The British Journal of Psychiatry, 118(545), 381-384.

[23] Tuchman, R., Cuccaro, M., \& Alessandri, M. (2010). Autism and epilepsy: historical perspective. Brain and Development, 32(9), 709718.

[24] Lebedyn, Z. (2019). The Use of Antidepressants by General Practitioners and Psychiatrists ( Personal Experience ).

[25] Lebedyn, Z. (2020). Antidepressants In General Practice And Psychiatry. European Journal of Medical and Health Sciences, 2(3). https://doi.org/10.24018/ejmed.2020.2.3.318

[26] Hedley, D., Uljarević, M., Wilmot, M., Richdale, A., \& Dissanayake, C. (2018). Understanding depression and thoughts of self-harm in autism: a potential mechanism involving loneliness. Research in Autism Spectrum Disorders, 46, 1-7.

[27] Tsarkov, A., \& Petlovanyi, P. The Role of Lamotrigine in the Treatment of Bipolar Depression.

[28] Delong, R. (2007). GABA (A) receptor alpha5 subunit as a candidate gene for autism and bipolar disorder: a proposed endophenotype with parent-of-origin and gain-of-function features, with or without oculocutaneous albinism. Autism, 11(2), 135-147.

[29] Munesue, T., Ono, Y., Mutoh, K., Shimoda, K., Nakatani, H., \& Kikuchi, M. (2008). High prevalence of bipolar disorder comorbidity in adolescents and young adults with high-functioning autism spectrum disorder: a preliminary study of 44 outpatients. Journal of affective disorders, 111(2-3), 170-175.

[30] Tsarkov, A., \& Petlovanyi, P. (2017). Pathological Gambling: The Old Problem of the Modern World. Imperial Journal of Interdisciplinary Research, 3(8), 216-221.

[31] Mazurek, M. O., Engelhardt, C. R., \& Clark, K. E. (2015). Video games from the perspective of adults with autism spectrum disorder. Computers in Human Behavior, 51, 122-130.

[32] Tsarkov, A., \& Petlovanyi, P. (2019). Neuropsychiatric Aspects of a Common Problem: Stroke. European Journal of Medical and Health Sciences, 1(3).

[33] Curtin, C., Jojic, M., \& Bandini, L. G. (2014). Obesity in children with autism spectrum disorders. Harvard review of psychiatry, 22(2), 93.

[34] Phiri, C., Tsarkov, A., Petlovanyi, P., \& Lingenda, G. Factors Contributing To Oral Diseases and Treatment Needs amongst Mental Patients at Chainama Hills College Hospital, Lusaka, Zambia.

[35] Blomqvist, M., Dahllöf, G., \& Bejerot, S. (2014). Experiences of dental care and dental anxiety in adults with autism spectrum disorder. Autism research and treatment, 2014. 